\title{
Determination of Critical Generator Group Using Accelerating Power and Synchronizing Power Coefficient in the Transient Energy Function Method
}

\begin{abstract}
Yeong-Han Chun ${ }^{\dagger}$
Abstract - This paper proposes an algorithm for determining critical generator lists using accelerating power and synchronizing power coefficient (SPC), and critical generator group (CGG) from CGG candidates, which is a combination of critical generators. The accurate determination of CGG provides a more accurate energy margin while providing system operator with information of possible unstable generator group. Classical transient energy function (TEF) method selects the critical generators with big corrected kinetic energy of each generator at the moment of fault removal. However, the generator with small acceleration after fault, that is, the generator with small corrected kinetic energy, is also likely to belong to CGG if the generator has small synchronizing power. The proposed algorithm has been verified to be effective compared with the classical TEF method. We utilized the power system of Korean Electric Power Corporation(KEPCO) as a test system.
\end{abstract}

Keywords: Transient stability, Critical generators, Transient energy function, Synchronizing power coefficient, Accelerating power, Mode of disturbance

\section{Introduction}

While investment in the construction of transmission network has been insignificant throughout the world, the electrical load has continued to grow. Therefore, it becomes necessary to improve operation technology through real-time security assessment. It is essential to continuously monitor whether stability can be maintained in the system for credible contingency by performing real-time evaluation of transient stability. Transient instability occurs when there is big disturbance in the system and is closely related to the size and nature of disturbance. The evaluation of transient stability aims to check whether the generator can maintain the synchronism against large disturbances like three line-to-ground faults.

Transient energy function (TEF) method is a very powerful transient stability analysis tool in terms of calculating speed compared with time stimulation method. Since the accurate calculation of transient energy and potential energy, and the accurate selection of critical generator group (CGG) affect the accurate evaluation of transient stability in TEF method, many studies have been conducted regarding these issues [1]-[4]. Some reports have posited that the accuracy of stability margin has improved through the introduction of the concept of corrected transient energy function. The accurate selection of $\mathrm{CGG}$ is the most fundamental issue for the evaluation of on-line transient stability.

The single machine equivalent (SIME) method [2], [3] is

\footnotetext{
$\dagger \quad$ Corresponding Author: Department of Electrical and Electronic Engineering, Hong-ik University, Korea (yhchun@hongik.ac.kr) Received: January 18, 2010; Accepted: December 20, 2010
}

also suggested as an evaluation method for on-line transient stability. Since SIME method uses time simulation method, which calculates rotor angles until CGG reaches instability, it determines in advance the CGG offline and uses it on-line. Therefore, it is very vulnerable to system environment. Classical TEF method prepares the candidate list of CGGs using kinetic energy. In this case, since CGG candidates are determined only with corrected kinetic energy, generators with small corrected kinetic energy and small synchronizing power are omitted from the CGG candidate lists.

\section{Classical Power System Model}

In this section, theoretical backgrounds of TEF method are overviewed [5].

The power into the network at node $i$, which is the electrical power output of machine $i$, is given by

$$
\begin{gathered}
P_{e i}=\operatorname{Re} \bar{E} \bar{I}^{*} . \\
P_{e i}=E_{i}^{2} G_{i i}+\sum_{\substack{j=1 \\
j \neq i}}^{n} E_{i} E_{j} Y_{i j} \cos \left(\theta_{i j}-\delta_{i}+\delta_{j}\right) \quad i=1,2, \cdots, n \\
=E_{i}^{2} G_{i i}+\sum_{\substack{j=1 \\
j \neq i}}^{n} E_{i} E_{j}\left[B_{i j} \sin \left(\delta_{i}-\delta_{j}\right)+G_{i j} \cos \left(\delta_{i}-\delta_{j}\right)\right](1) \\
=E_{i}^{2} G_{i i}+\sum_{\substack{j=1 \\
j \neq i}}^{n}\left[C_{i j} \sin \left(\delta_{i}-\delta_{j}\right)+D_{i j} \cos \left(\delta_{i}-\delta_{j}\right)\right]
\end{gathered}
$$


where $C_{i j}=E_{i} E_{j} B_{i j}$ and $D_{i j}=E_{i} E_{j} G_{i j}$.

In eq. (1), the admittance matrix terms used must correspond to the network conditions for which the electrical power $P_{e i}$ is computed. If the disturbance is a fault, prefault, faulted, and post-fault networks have different $\bar{Y}$ matrices. The swing equations of generators are given by the following:

$$
\frac{2 H_{i}}{\omega_{R}} \frac{d \omega_{i}}{d t}+D_{i} \omega_{i}=P_{m i}-E_{i}^{2} G_{i i}+\sum_{\substack{j=1 \\ j \neq i}}^{n} E_{i} E_{j} Y_{i j} \cos \left(\theta_{i j}-\delta_{i}+\delta_{j}\right)_{(2)}
$$

Where $H_{i}$ is the inertia constant for generator $i$ in Mws/MVA and $\omega_{i}$ and $\omega_{R}$ is the speed of the generator and the reference speed, respectively.

\subsection{Center of Inertia (COI) Formulation}

In the eq. (2), the generator's angles and speeds are given with respect to a synchronous frame of reference. The COI is defined as follows:

$$
\begin{aligned}
& \delta_{0}=\frac{1}{M_{T}} \sum_{i=1}^{n} M_{i} \delta_{i} \\
& \dot{\delta}_{0}=\frac{1}{M_{T}} \sum_{i=1}^{n} M_{i} \dot{\delta}_{i}
\end{aligned}
$$

Where $M_{i}=$ inertia constant for generator; $i=2 H_{i} / \omega_{R}$, and $M_{T}=\sum_{i=1}^{n} M_{i}$.

The motion of the COI is defined by the following equation:

$$
M_{T} \dot{\omega}_{0}=\sum_{i=1}^{n} P_{m i}-P_{e i}=\sum_{i=1}^{n} P_{i}-\sum_{i=1}^{n-1} \sum_{j=i+1}^{n} D_{i j} \cos \delta_{i j}=P_{C O I}
$$

Where $P_{i}=P_{m i}-E_{i}^{2} G_{i i} \quad i=1,2, \cdots, n$.

The generators' angles and speeds relative to the $\mathrm{COI}$ are defined by the following:

$$
\begin{aligned}
& \theta_{i}=\delta_{i}-\delta_{0} \\
& \widetilde{\omega_{i}}=\dot{\delta}_{i}-\dot{\delta}_{0}
\end{aligned}
$$

The motion equation of the system becomes the following:

$$
\begin{aligned}
& M_{i} \dot{\tilde{\omega}_{i}}=P_{m i}-P_{e i}-\frac{M_{i}}{M_{T}} P_{C O I} \\
& \dot{\theta}_{i}=\widetilde{\omega}_{i} \quad i=1,2, \cdots, n .
\end{aligned}
$$

The COI variables satisfy the equality constraints, as shown below:

$$
\sum_{i=1}^{n} M_{i} \dot{\theta}_{i}=\sum_{i=1}^{n} M_{i} \widetilde{\omega_{i}}=0
$$

Eq. (6) represents the dynamics of the post-disturbance system.

\subsection{Corrected Kinetic Energy}

The kinetic energy associated with the gross motion of a group of $\mathrm{k}$ machines having angular speeds $\widetilde{\omega_{1}}, \widetilde{\omega_{2}}, \widetilde{\omega_{3}}, \cdots \widetilde{\omega_{k}}$ is the same as the kinetic energy at COI. The speed of the inertial center of that group and its kinetic energy are given by the following:

$$
\begin{gathered}
\widetilde{\omega_{c r}}=\sum_{i=1}^{k} M_{i} \widetilde{\omega_{i}} / \sum_{i=1}^{k} M_{i} \\
V_{K E_{c r}}=\frac{1}{2}\left[\sum_{i=1}^{k} M_{i}\right]\left(\widetilde{\widetilde{\omega}_{c r}}\right)^{2}
\end{gathered}
$$

The disturbance splits the generators of the system into two groups: the critical generators and the rest of the generators. The two groups' inertial centers have inertia constants and angular speeds $M_{c r}$ and $\omega_{c r}$, and $M_{s y s}$ and $\widetilde{\omega_{s y s}}$, respectively. The gross motion of the two groups approximates that of a two-machine system. The kinetic energy causing the separation of the two groups is the same as that of an equivalent one-machine infinite-bus (OMIB) system having inertia constant $M_{e q}$ and angular speed $\widetilde{\omega_{e q}}$, given by the following equation:

$$
\begin{aligned}
M_{e q} & =\frac{M_{c r} \cdot M_{s y s}}{M_{c r}+M_{s y s}} \\
\widetilde{\omega_{e q}} & =\widetilde{\omega_{c r}}-\widetilde{\omega_{s y s}}
\end{aligned}
$$

In addition, the corresponding kinetic energy is as follows:

$$
V_{K E \mid c o r r}=\frac{1}{2} M_{e q}\left(\widetilde{\omega_{e q}}\right)^{2}
$$

\subsection{Energy Margin and Transient Stability Assess- ment}

The transient energy function is defined by the following;

$$
V=\frac{1}{2} M_{e q}\left(\widetilde{\omega_{e q}}\right)^{2}-\sum_{i=1}^{n} P_{i}\left(\theta_{i}-\theta_{i}^{s 2}\right)
$$




$$
-\sum_{i=1}^{n-1} \sum_{j=i+1}^{n}\left[C_{i j}\left(\cos \theta_{i j}-\cos \theta_{i j}^{s 2}\right)-\int_{\theta_{i}^{s 2}+\theta_{j}^{s}}^{\theta_{i}+\theta_{j}} D_{i j} \cos \theta_{i j} \cdot d\left(\theta_{i}+\theta_{j}\right)\right]
$$

Where $\theta_{i}^{s 2}$ is the post-disturbance stable equilibrium point.

The transient energy margin is then defined as follows:

$$
\Delta V=V_{c r}-V_{c l}
$$

Where $V_{c r}$ is the critical energy, which is the potential energy at the controlling unstable equilibrium point (UEP), and $V_{c l}$ is the transient energy at the end of disturbance. Substituting for $V_{c r}$ and $V_{c l}$ from eq. (11), we have the following:

$$
\begin{aligned}
\Delta V= & -\frac{1}{2} M_{e q} \tilde{\omega}_{e q}^{c l^{2}}-\sum_{i=1}^{n} P_{i}\left(\theta_{i}^{u}-\theta_{i}^{c l}\right) \\
& -\sum_{i=1}^{n-1} \sum_{j=i+1}^{n}\left[C_{i j}\left(\cos \theta_{i j}-\cos \theta_{i j}^{s 2}\right)\right. \\
& \left.-D_{i j} \frac{\theta_{i}^{u}-\theta_{i}^{c l}+\theta_{j}^{u}-\theta_{j}^{c l}}{\left(\theta_{i j}^{u}-\theta_{i j}^{c l}\right)}\left(\sin \theta_{i j}^{u}-\sin \theta_{i j}^{c l}\right)\right]
\end{aligned}
$$

The degree of stability can be obtained if $\Delta V$ is normalized with respect to the corrected kinetic energy at the end of disturbance.

$$
\Delta V_{n}=\frac{\Delta V}{V_{K E \mid c o r r}}
$$

\section{Determination of CGG}

The decision on the correct controlling UEP is very important for the exact calculation of the energy margin. As CGG is a set of generators tending to separate from the system, generators with advanced angles at the fault clearing instance are considered to be critical generator candidates [10]. Generators with small advanced angles at the moment of fault clearing can have small synchronizing power. As generators with small synchronizing power also tend to separate from the system, such generators should be included as CGG candidates.

\subsection{Configuration of OMIB}

Transient stability refers to the problem of dynamic interaction of transient energy between two generator groups: the generator group tending to separate from the system and the rest of the generators.

Generators that belong to each group can be reduced to one-machine equivalent generator, as shown in eqs. (15) and (16), using the principle of COI. The two coherent generator groups can be reduced into one-machine equiva- lent generator using each weighted average.

$$
\begin{array}{cc}
M_{C R}=\sum_{k \in C R} M_{k} \quad \delta_{C R}=M_{C R}^{-1} \sum_{k \in C R} M_{k} \delta_{K} \\
M_{S Y S}=\sum_{k \in S Y S} M_{k} & \delta_{S Y S}=M_{S Y S}^{-1} \sum_{k \in S Y S} M_{k} \delta_{K}
\end{array}
$$

Where M: generator inertia coefficient

$\delta$ : phase angle of generator

$\mathrm{k}$ : index of generator

CR: a set of generators tending to separate from the system

SYS: a set of generators remaining stable in the system

Since the reduced system consists of two incoherent generators, the generator phase angle, inertia coefficient, mechanical input, and electric output of the OMIB system must be defined in order to reduce these two incoherent generators into an OMIB system.

$$
\begin{gathered}
\delta=\delta_{C R}-\delta_{S Y S} \\
M=\frac{M_{C R} M_{S Y S}}{\left(M_{C R}+M_{S Y S}\right)} \\
P_{m}=M\left(M_{C R}^{-1} \sum_{k \in C R} P_{m k}-M_{S Y S}^{-1} \sum_{j \in S Y S} P_{m j}\right) \\
P_{e}=M\left(M_{C R}^{-1} \sum_{k \in C R} P_{e k}-M_{S Y S}^{-1} \sum_{j \in S Y S} P_{e j}\right)
\end{gathered}
$$

Where

$P_{m}:$ mechanical input of generator
$P_{e}:$ electrical output of generator

Accordingly, if the acceleration in the OMIB system is expressed as $P_{a}$, the dynamic feature for the OMIB system can be expressed as swing equation:

$$
M \dot{\delta}=M \omega=P_{m}-P_{e}=P_{a}
$$

Where

$$
\begin{aligned}
& \omega: \text { angular velocity of generator } \\
& P_{a}: \text { acceleration power of generator }
\end{aligned}
$$

\subsection{Ratio of Accelerating Power (RAS) to Synchro- nizing Power Coefficient (SPC)}

SPC [8] at angle $\delta_{0}$ is defined by the following:

$$
S P C=\left.\frac{\partial P_{e}}{\partial \delta}\right|_{\delta=\delta_{0}}=\frac{V_{1} V_{1}}{X} \cos \delta_{0}=P_{\max } \cos \delta_{0}
$$

$P_{\max }$ can be interpreted as a degree of strength with which the generator is connected to the system. The deceleration of the machine is dependent on the SPC and inertia $\mathrm{H}$. Therefore, the SPC at point $\mathrm{G}$ and the accelerating power $P_{e A}-P_{e B}$ can be an excellent index showing how the machine is tending to separate from the system. The 


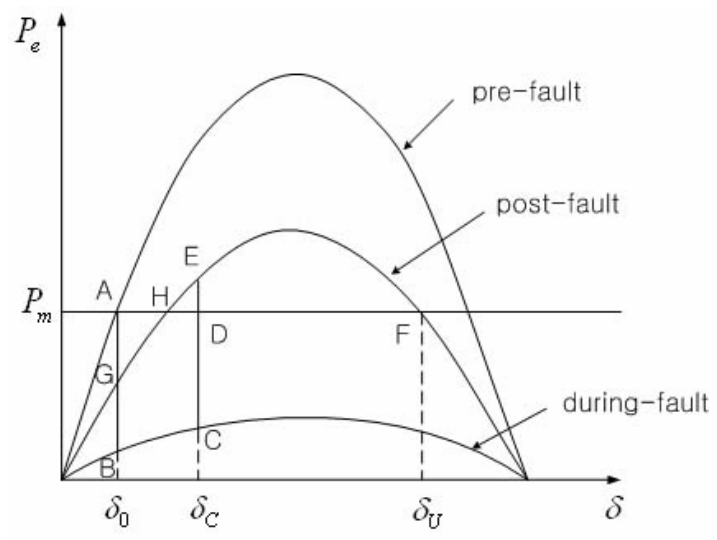

Fig. 1. Equal area criterion of the OMIB system.

RAS is defined by the ratio of the accelerating power to the $\mathrm{SPC}$, as in eq. (23).

$$
R A S=\frac{P_{e A}-P_{e B}}{S P C_{G}}
$$

\subsection{Selection of Candidate Generators for the Selec- tion of CGG Using RAS}

To calculate the RAS as described in eq. (23), this study created as many OMIB as the number of generators and calculated the RAS for each case by assuming each generator as a critical generator and the others as the stable generator group. This led to improved results compared with the method that uses only acceleration.

The procedure to determine the candidates of CGG using the RAS is given as follows.

(1) Determine the system voltage, generator output, and generator phase angle from the power flow calculation.

(2) On fault condition, reduce the system to OMIB system for each generator and calculate the accelerating power for each generator while the phase angle of generator is fixed.

(3) On post-fault condition, reduce the system to OMIB system for each generator and calculate the SPC for each generator while the phase angle of generator is fixed.

(4) Arrange the RAS of the generators in descending order.

(5) Determine the generators with the RAS over the boundary condition. The boundary condition is determined based on the engineering sense. These generators constitute critical generators list that can be candidates for $\mathrm{CGG}$.

(6) Construct the candidate CGGs by the combination of generators from the critical generators list. If some generators of the same type are placed at the same site and connected to the same bus, the generators are considered a group. For example, considering the case when eight generators are in the critical generators list, generators $\{1,2\}$ and $\{3,4,5,6\}$ are of the same type and placed at the same site and connected to the same bus, respectively. As such, 15 CGG candidates can be selected from the critical generators list as follows.

Critical generators list $=\{\{1,2\},\{3,4,5,6\}, 7,8\}$ CGG candidates $=\{1,2\},\{3,4,5,6\},\{7\},\{8\},\{1$, $2,3,4,5,6\},\{1,2,7\},\{1,2,8\},\{3,4,5,6,7\}$, $\{3,4,5,6,8\},\{7,8\},\{1,2,3,4,5,6,7\},\{1,2,3$, $4,5,6,8\},\{1,2,7,8\},\{3,4,5,6,7,8\},\{1,2,3$, $4,5,6,7,8\}$

(7) Among the CGG candidates, the one with the lowest normalized potential energy margin is selected as CGG. This CGG decides the controlling UEP.

$$
\Delta V_{P E \mid n}=\frac{V_{P E}}{V_{K E \mid c o r r}}
$$

Where

$$
\begin{aligned}
& \Delta V_{P E}=V_{P E}^{u}-V_{P E}^{c l} \\
&=-\sum_{i=1}^{n} P_{i}\left(\theta_{i}^{u}-\theta_{i}^{c l}\right)-\sum_{i=1}^{n-1} \sum_{j=i+1}^{n}\left[C_{i j}\left(\cos \theta_{i j}-\cos \theta_{i j}^{s 2}\right)\right. \\
&\left.-D_{i j} \frac{\theta_{i}^{u}-\theta_{i}^{c l}+\theta_{j}^{u}-\theta_{j}^{c l}}{\left(\theta_{i j}^{u}-\theta_{i j}^{c l}\right)}\left(\sin \theta_{i j}^{u}-\sin \theta_{i j}^{c l}\right)\right]
\end{aligned}
$$

\subsection{Algorithm for Determining CGG}

The sequence for determining CGG is as follows.

(1) Load the input data required for the evaluation of stability. Input data include the accident removal time, accident bus and line, inertia integer of generator, and input data required for the calculation of power flow.

(2) Solve the power flow equation and carry out network reduction. At this time, reduce and remove other buses except the generator bus, and add the loads to the diagonal term of the Y-Matrix using the positive sequence impedance model. In addition, configure the final Y-Matrix by considering the impedance of generator internal bus.

(3) Calculate the COI phase angle for each generator. That is, determine the phase angle of the COI for one generator and for all the remaining generators. This is the preliminary procedure of the following process that configures OMIB by assuming each one as advance generator.

(4) Configure OMIB for each generator using the abovementioned results.

(5) Calculate the RAS index, the index of acceleration and SPC, and arrange the generators in descending order.

(6) Designate the generators up to the 12th place among the arranged generators as the candidates of critical generators.

(7) Obtain the energy margins of the CGG candidates 
Table 1. Comparison of CGG among time simulation (PSS/E), classical TEF, and RAS

\begin{tabular}{|c|c|c|c|c|c|c|}
\hline \multirow{2}{*}{ No. } & \multicolumn{3}{|c|}{ Contingency } & \multirow{2}{*}{\begin{tabular}{|c|}
$\begin{array}{c}\text { Time simulation } \\
(\mathrm{PSS} / \mathrm{E})\end{array}$ \\
$\mathrm{CGG}$
\end{tabular}} & \multirow{2}{*}{$\frac{\text { Classical TEF }}{\text { CGG }}$} & \multirow{2}{*}{$\begin{array}{c}\text { CGG obtained } \\
\text { by RAS } \\
\text { CGG }\end{array}$} \\
\hline & From & To & CKT & & & \\
\hline 1 & 1500 & 5155 & 1,2 & $\begin{array}{l}25151,25152 \\
25153,25154\end{array}$ & $\mathrm{~N} / \mathrm{A}$ & $\begin{array}{l}25151,25152 \\
25153,25154\end{array}$ \\
\hline 2 & 3600 & 4400 & 1 & $\begin{array}{l}24451,24452 \\
24453,24454\end{array}$ & $\begin{array}{l}24451,24452 \\
24453,24454\end{array}$ & $\begin{array}{l}24451,24452 \\
24453,24454\end{array}$ \\
\hline 3 & 4010 & 6030 & 1,2 & $\begin{array}{l}26101,26102 \\
26103,26104 \\
26105,26106 \\
26201,26202 \\
26203,26204\end{array}$ & $\begin{array}{l}26201,26202 \\
26203,26204\end{array}$ & $\begin{array}{l}26101,26102 \\
26103,26104 \\
26105,26106 \\
26201,26202 \\
26203,26204\end{array}$ \\
\hline \multirow{2}{*}{4} & \multirow{2}{*}{5500} & 8500 & 1 & \multirow{2}{*}{$\begin{array}{l}25151,25152 \\
25153,25154\end{array}$} & \multirow{2}{*}{$\begin{array}{l}25151,25152 \\
25153,25154\end{array}$} & \multirow{2}{*}{$\begin{array}{l}25151,25152 \\
25153,25154\end{array}$} \\
\hline & & 8501 & 2 & & & \\
\hline 5 & 6300 & 6900 & 1,2 & $\begin{array}{l}26101.26102 \\
26103.26104 \\
26105.26106\end{array}$ & $\begin{array}{l}26101,26102 \\
26103,26104 \\
26105,26106 \\
26201,26202 \\
26203,26204\end{array}$ & $\begin{array}{l}26101,26102 \\
26103,26104 \\
26105,26106 \\
26201,26202 \\
26203,26204\end{array}$ \\
\hline \multirow{2}{*}{6} & \multirow{2}{*}{6450} & 7155 & 1 & \multirow{2}{*}{27155,27156} & \multirow{2}{*}{27155,27156} & \multirow{2}{*}{27155,27156} \\
\hline & & 7156 & 2 & & & \\
\hline \multirow{2}{*}{7} & 6600 & 7100 & 2 & $\begin{array}{l}27151,27152 \\
27153,27154\end{array}$ & N/A & 27153,27154 \\
\hline & & 7101 & 1 & 27155,27156 & & \\
\hline 8 & 7101 & 7156 & 1 & 27155,27156 & 2715527156 & 2715527156 \\
\hline & 7100 & 7155 & 2 & & & \\
\hline 9 & 7101 & 7156 & 2 & 2715527156 & 2715527156 & 2715527156 \\
\hline & 7100 & 7155 & 1 & & & \\
\hline & 7100 & & 1 & & 27151,27152 & \\
\hline 10 & 7101 & 7600 & 2 & $\begin{array}{l}27151,27152 \\
27153,27154 \\
27155,27156\end{array}$ & $\begin{array}{l}27155,27156 \\
27251,27252 \\
27271,27272 \\
27751,27752 \\
27753,27754 \\
27755,27756\end{array}$ & $\begin{array}{l}27151,27152 \\
27153,27154 \\
27155,27156\end{array}$ \\
\hline & 7100 & & 1 & & $\begin{array}{l}27151,27152 \\
2715327154\end{array}$ & \\
\hline 11 & 7101 & 7900 & 2 & $\begin{array}{l}27151,27152 \\
27153,27154 \\
27155,27156\end{array}$ & $\begin{array}{l}27155,27156 \\
27251,27252 \\
27271,27272 \\
27751,27752 \\
27753,27754 \\
27755,27756\end{array}$ & $\begin{array}{l}27151,27152 \\
27153,27154 \\
27155,27156\end{array}$ \\
\hline 12 & 7101 & 7900 & 2 & 2715527156 & 27156 & 2715527156 \\
\hline & 7100 & & 1 & & 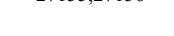 & 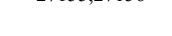 \\
\hline 13 & 7500 & 7600 & 1,2 & $\begin{array}{l}27151,27152 \\
27153,27154 \\
27155,27156\end{array}$ & $\begin{array}{l}27151,27152 \\
27153,27154\end{array}$ & $\begin{array}{l}27151,27152 \\
27153,27154 \\
27155,27156\end{array}$ \\
\hline 14 & 7900 & 10401 & 1 & $\begin{array}{l}27251,27252 \\
27271,27272 \\
27751,27752 \\
27753,27754 \\
27755,27756\end{array}$ & $\begin{array}{l}27251,27252 \\
27271,27272 \\
27751,27752 \\
27753,27754 \\
27755,27756\end{array}$ & $\begin{array}{l}27251,27252 \\
27271,27272 \\
27751,27752 \\
27753,27754 \\
27755,27756\end{array}$ \\
\hline 15 & 8156 & 8250 & 1 & 28154 & 28154 & 28154 \\
\hline & 8155 & & 2 & & & \\
\hline 16 & 8155 & 8250 & 2 & 28151,28152 & 28151,28152 & 28151,28152 \\
\hline & 8156 & & 1 & 28153 & 28153 & 2815 \\
\hline 17 & 8156 & 8500 & 1 & 28154 & 28154 & 28154 \\
\hline & 8155 & 8501 & 2 & & & \\
\hline 18 & 9350 & 9501 & 1,2 & $\begin{array}{c}29351,29352 \\
29353,29365 \\
29366\end{array}$ & $\begin{array}{c}29351,29352 \\
29353\end{array}$ & $\begin{array}{c}29351,29352 \\
29353\end{array}$ \\
\hline & 9500 & & 1 & & & 29251,29252 \\
\hline 19 & 9501 & 10800 & 2 & N/A & N/A & $\begin{array}{c}29353,30152 \\
30153,30154 \\
30155\end{array}$ \\
\hline 20 & 10150 & 10700 & 1,2 & $\begin{array}{l}30151,30152 \\
30153,30154 \\
30155,30156\end{array}$ & $\begin{array}{l}30151,30152 \\
30153,30154 \\
30155,30156\end{array}$ & $\begin{array}{l}30151,30152 \\
30153,30154 \\
30155,30156\end{array}$ \\
\hline
\end{tabular}

obtained above and select the CGG with the lowest normalized energy margin as the CGG.

The candidates of critical generators are selected up to the 12 th place because time simulation results show that the generators tending to separate from the system do not exceed the 12 generators in the KEPCO system. If the candidate generator is designated up to the 12th place and the generators with the same type, located at the same site, and connected to the same generator bus are assumed as one generator group, the number of critical generators can be reduced to three to five. If the number of critical generators is 3 , then the number of CGG candidates is 7 ; consequently, if the number of critical generators is 5 , the number of CGG candidates is 31 .

\section{Application Results}

To verify the proposed algorithm, the KEPCO system was used for the application. The severity of line accident may differ depending on the location of the fault. The contingency of three-phase-to-ground fault and removal of the faulted line was assumed in the study.

Table 1 shows the comparative results of selecting CGG by different methods: PSS/E time simulation, classical TEF method, and the proposed method. In the first and seventh cases, classical TEF method could not determine the appropriate CGG, whereas the proposed method generated the same CGG as the PSS/E time simulation method. The classical TEF method produced different CGG as that of the proposed and PSS/E time simulation methods, especially in cases 3, 10, and 11 .

\section{Conclusion}

The concept of RAS was proposed for fast selection of critical generators, which is the most important procedure in selecting the accurate CGG, and thus the accurate control of UEP. The proposed algorithm for selecting CGG was applied to the KEPCO system, and we observed that it has better performance compared with the classical TEF method.

\section{Acknowledgment}

This work was supported partly by the Power IT Research Program of the Ministry of Knowledge and Economy, Republic of Korea and party by Hongik university research fund.

\section{References}

[1] Y. Zang, L. Wehenkel, M. Pavella, "SIME : A Com- 
prehensive Approach to Fast Transient Stability Assessment," Tran of IEE Japan, Vol. 118-B, No. 2, pp. 127-132, 1998

[2] Y. Zang,L. Wehenkel, P. Rousseaux, M. Pavella, "SIME : A Hybrid Approach to Fast Transient Stability Assessment and Contingency Selection," Electric Power \& Energy System. Vol.19, No.3, pp.195-208. 1996.

[3] Edward Wilson Kimbark, Power System Stability, John Wiley \& Sons, Inc. 1947

[4] A. A. Fouad, Vijay Vittal, Power System Transient analysis Using the Transient Energy Function Method, Prentice Hall 1992

[5] A. L. Bettiol, Y. Zhang, L. Wehenkel, M. Pavella, "Transient stability investigations on a Brazilian network by SIME," Advances in Power System Control, Operation and Management, 1997. APSCOM-97. Fourth international conference on (Conf. Publ. No. 450) Volume 1, 11-14 pp. L1 - L6, Nov. 1997

[6] A. A. Fouad, Vijay Vittal "Power System Transient Stability Using Individual Machine Energy Function," IEEE Tran on Circuit and Systems, vol. CAS30, NO. 5, May 1983

[8] P. M. Anderson, A. A. Fouad, Power System Control and Stability," John Wiley \& Sons, Inc.
[9] B. Lee, S.H. Kwon, J. Lee, H.K. Nam, J.B. Choo, D. H. Jeon, "Fast Contingency Screening for On-line Transient Stability Monitoring and Assessment of the KEPCO System," Generation, Transmission and Distribution, IEE Proceedings-Volume 150, Issue 4, pp. 399-404, 14 July 2003.

[10] Ribbens-Pavella, P. G. Murthy, and J. L. Horward, "The Acceleration Approach to Practical Stability Domain Estimation in Power Systems", In Proceedings 20th IEEE Conference on Decision and Control, pp. 471-477, 1981.

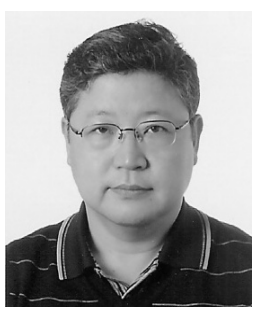

Yeong-Han Chun received his B.S. and M.S. degrees from Seoul National University, Korea in 1983 and 1985, respectively, and his Ph.D. degree from Tokyo University, Japan in 1997. He was with the Korea Electro-technology Research Institute (KERI) from 1985 to 2002. He then joined Hongik University, where he is currently an Associate Professor of Electrical Engineering. His research interests are power system control and stability. 